\title{
Review of: "Exploration On Definition And The Components of Food And Nutrition Literacy Among Junior Secondary School Students: A Qualitative Study"
}

\author{
Mohammed Reza Shadmand Foumani Moghadam
}

Potential competing interests: The author(s) declared that no potential competing interests exist.

In this study, the authors exploring on definition and understanding components of food and nutrition literacy in Junior Secondary School Students. the study was well described and can have essential reginal effects. The method and analysis of study was normal. However the using of voice recording and the interviewing method was a great idea that can increase the quality and reliability of the current study. Table 3 is the main strength and finding of the study and researchers. other researchers and developers can use that for further development of the current tool. There will be great achievement if the authors continue researching on this field and improve a questionnaire (a semi-qualitive questionnaire) with a scoring system to categorize the FANLit of individuals in the future.

However, there are some required improvements. The English language required to be revised. There are some grammatical and word choosing errors in the text. It is not smooth and can make compilation for readers. These language complications can effect and decrease the quality of the study.

if the authors make an major revision to the current study, it would have a good potential to receive considerable citations in the future.

There are some other comments:

\section{Minor}

When we start sentence, we use word form of numbers. In abstract-method-line 2, 49 Should be changed to fourteen nine

The conclusion of the abstract is better to conclude findings of the study better with more details.

The last part of introduction paragraph 2 "While food provides energy, nutrients affect health[13]". What do you try to transfer? Does you want to say "food provides energy, nutrients that affect health"? And for "while' what are you comparing this word to? It is hard to understand In setting mention percentage for the prevalence's. or any other index that was used.

\section{Major}

The text required several grammatical and language improvements. In few part it is hard to follow. But 
overall it is well written.

In recruiting and sampling, you divided participants to 4 groups. What was the critia of this division? How you decide to put them in each of the groups? OR are you taking about the stages of your sampling? I find the answer in the table but at the first look it is confusing. Make it clear in the text please About the number of panel reviewers; have you statically checked the number of each panel? Or you used the available experts? Or the reviewer you choice is as reliable as reference (some one with great background and publication history in the field)? You should aware that in normal condition, 1-3 reviewer is not reliable for qualities assessments.

The discussion is good but can be written better. However, works on FANLit is limited that make discussing a bit harder

Is all the limitations and strengths of the study are qualitative approve and specic context of the subjects? What about sample size of panels, the recording and trustworthiness checking, and the nature of these kind of studies? In addition what is your suggestion for further studies? Open a path to research for further works 